While cysts of this type are often found in the region of the eye the interest of this case lies, first, in the fact that it must have arisen posterior to the eyeball, and secondly, in the size to which it has grown causing complete flattening of the eyeball as seen in Figs. 3 and 4.

In the literature on tumours of the orbit to which I have had access I have not been able to find a case similar to this one.

I wish to express my thanks to Dr. Garven for the notes on the pathological examination and also to $\mathrm{Mr}$. Li Hung Hsin for the drawings.

\title{
TRACHOMA IN THE INDIANS OF WESTERN CANADA
}

\author{
BY \\ DR. J. J. WALL \\ DEPARTMENT OF INDIAN AFFAIRS, CANADA
}

THE trachoma situation in the Indians of Western Canada constitutes a serious problem when viewed from the economic, humanitarian and public health standpoints. To form an adequate conception of the visual devastation wrought by this disease together with its complications and sequelae, one should really visit some of the Western Settlements.

The total economic loss resulting from the ravages of the disease, is difficult to compute at the present time. Many Indians are in their transitional period in various parts of the West. Hunting and trapping as a sole source of livelihood for these people has largely disappeared, being replaced by agriculture and stock raising on Reserves placed aside for their use. Civilization with all its resultant obligations and dangers to these peoples has definitely supplanted the old régime. Huts and houses, frequently overcrowded and usually badly ventilated in the colder weather, have replaced the admirable teppee and tent. Every effort must be expended to check trachoma and the economic loss engendered by the disorder in those individuals whose ultimate disability precludes any possibility of self-support. Failing this, contributions must be forthcoming from the public treasury toward such an individual's support which would undoubtedly constitute a considerable financial outlay by the country in the future.

One must take cognizance of the humanitarian aspect of this malady. The suffering of many of these afflicted individuals is most intense, due to the chronic or sub-acute inflammation of the eyelids and also to the accompanying iritis. The latter, especially, 
is present throughout the summer months when the intense heat, fine alkali dust and sand storms aggravate the condition. During this period a sub-chronic or chronic stage advances to that of a sub-acute condition. The constant spasm of the orbicularis muscle, the photophobia, the lacrymation and congestion of the ocular vessels, convey to the observer an intimation of the pain and discomfort of the individual.

Trachoma is not confined to the Indians alone. Many cases can be noted in immigrants of Eastern and South-Eastern European extraction. Similar cases have been seen in the Chinese of British Columbia. Many people of mixed Indian and white blood are afflicted with the disorder. Non-amenable to administration and regulation, as are the Indians under their agent, these folk frequently wander from place to place during such periods as they are not settled adjacent to a Reserve. A certain element of these half-breeds unfortunately consider themselves far superior to either the white or Indian element. It is feared that any measures instituted to eradicate this communicable disorder or control the wanderings of these people will be met with little enthusiasm and even passive opposition.

Cases of trachoma in the Indians are distributed from Ontario westward through the Prairie Provinces and into British Columbia. Ontario has the lowest incidence, the greatest occurring in the central and southern parts of the prairies together with the eastern and central portion of British Columbia. In this area at least 70 per cent. of cases of markedly impaired vision or incurable blindness is due to this malady, together with the complications and sequelae. In certain settlements the incidence of infected individuals may reach 40 to 50 per cent. of the population. The northern portions of Alberta, Manitoba and the Pacific coastal region are the least affected. Cases disclosed in these latter areas are of a much more benign character than those encountered in the trachoma "belts" of the prairies and British Columbia.

Factors contributing to the high incidence and severity of cases are high altitude, finely pulverised alkali soil, frequent sand and dust storms, intensive heat and extreme dryness of the summer season. Settlements where these conditions prevail, where housing conditions are poor due to overcrowding, where there is a lack of individual and personal hygiene, invariably demonstrate the highest percentage of blindness. Fine alkali dust and sand blown by the high winds into the eyes of trachoma patients aggravate the already-present conjunctivitis which is further increased by rubbing with the hands and fingers. Small abrasions are formed in the cornea by the fine scarifying bodies. The superimposed irritation leads to a more rapid growth of the trachomatous pannus downward through the cornea. The possibility of corneal ulcers 
increases with their ever present danger of either perforation of the globe or subsequent formation of thick corneal opacities which in many cases are prone to cover the pupillary area.

Serious impairment of vision can usually be attributed to one or a combination of the following causes :-

(a) Perforation of the globe due to trachomatous ulcer which may permit some of the eye contents to escape. The end result of this is a sightless, shrunken eyeball.

(b) Dense opacity of the cornea from trachomatous pannus. Infiltrating from above downwards, this process advances into the cornea accompanied by numerous arborescent vessels. The remarkable smoothness, lustre and transparency of the normal structure is converted to a rough, dull opaque structure somewhat similar to that of irregularly heavily-frosted glass. The ultimateappearance of the eyeball is that of a boiled onion.

(c) Diffuse corneal opacities from trachomatous ulcers. When situated in the area of the pupil these may greatly limit or even totally prevent the transmission of visual stimuli to the retina.

(d) Secondary glaucoma from the formation of plastic exudates with consequent blocking of the filtration angle.

The general Indian conception of the disorder is not that of a communicable disease. Many believe the process of losing their sight is concomitant with greying hair and other manifestations of age changes, impossible to be avoided. These cases correspond to the type which have slowly and progressively lost their sight by pannus advancing into the cornea. Others who have been blinded by the more fulminating process of a perforating ulcer claim this disaster was due to a foreign body which entered the eye at any time from a few minutes to a few hours prior to the calamity. Others with a badly damaged cornea due to pannus and opacities explain their condition as the direct result of being bewitched by an enemy or conjuror, by means of a spell or a charm especially designed against the victim. None of these patients will ever surmise the identity of the responsible party. The belief holds that divulging the name of the sorcerer will be followed by still more dire consequences. The only relief to be obtained is through the offices of a necromancer, who will prescribe some medicine or charm which will remove the sand which had been originally conjured into the eye.

Many cases of trachoma noted in the children are undoubtedly attributable to the old grandmother occupying the same house. These old people are very strongly attached to the young children, especially the grandsons. The usual head dress constantly worn by these old people is a large silk kerchief. This is worn over the head in a manner very similar to that seen in the peasants of Eastern Europe. The scarf is tied under the chin with a large 
knot, the loose ends of which hang downwards. The loose ends are always utilized to mop away the continuous secretion from the diseased eyes which constantly overflows the cheeks. It serves a dual, but dangerous, purpose to wipe the nose and cheeks of the child which she so frequently coddles. It is not surprising that many cases are noted in young children.

The general lack of even elementary and individual hygiene in many of the Indian houses plays an important rôle in the dissemination of trachoma. The common towel and wash basin utilized by a whole family and their visiting guests is a frequent sight. In a household harbouring an advanced case with profuse secretion, it is not uncommon to note a majority of the other members afflicted with the disorder in varying stages.

It has been occasionally suggested that trachoma is caused solely by nutritional disturbances and unbalanced diet. Observations made with an impartial mind demonstrate that this is a ridiculous and untenable conception. It is quite certain that, all other factors being equal, a patient with a scrofulous diathesis or suffering from mal-nutrition tends to run a more malignant and refractory course. In such cases phlyctenular conjunctivitis is not infrequently an accompanying disorder. Scrofulous cases treated with copper citrate ointment or copper sulphate pencil for four months demonstrate a response similar to that noted in a more healthy individual with similar medication for a six weeks' period. To supplement all ocular therapeutics, an abundant diet is recommended, especially for those cases in the residential schools. The importance of this auxiliary measure in the eradication of this disorder in the young is constantly stressed. Mal-nutrition or avitaminosis when represented as the sole causative factor in trachoma must be rejected as a most dangerous theory.

The exact causative organism of trachoma has not been definitely established, although great credit is due to Lindner, Prowaczek and Noguchi for their efforts in this field. Whatever the exact aetiological factor may be, it is important to stress the part played by the pathological eye discharge in spreading the disorder. The danger of an individual to a community is in direct proportion to the amount of lacrymal secretion. A trachoma patient with complicating corneal ulcer and profuse discharge is especially dangerous as an agent of dissemination.

The possibility of the Indian introducing the disease into the white population must not be overlooked. If the Indian Reserve were an entirely self-supporting economic unit and the Indian confined entirely to his settlement, little danger might be anticipated by the surrounding white element. The Indian population is increasing. Over population will ultimately force, and is forcing in some areas, the individual to secure a supplementary living from 
labour in the surrounding territory. Many Indians augment their living on the Reserve by temporary employment with surrounding farmers. Others are engaged in fishing, canning, hop-picking, and cattle round-ups. Some of the girls secure employment as domestics in homes and hotels, others marry whites and leave the Reserve. The danger of positive cases introducing their disease into those workmen with whom they fraternise and associate is very apparent. Especially true is this in places of congregation such as bunkhouses where the roller or common towel, wash basin and soap would prove most important links in the chain of dissemination. Although my work is confined almost entirely to Indians, cases have been noted in the whites which undoubtedly had their origin in the native population. The Indian is no greater menace today than is the person of mixed blood or half-breed. As time progresses and more residents of a Reserve seek employment amongst the whites, the danger will be correspondingly increased.

A rancher of British Columbia had contracted the disorder from Indians whom he had employed in a cattle round-up a few years ago. On examination the lids were hypertrophied, granular and intensely red. Pannus with vascularization had advanced downward into the cornea. Fine vessels were present over the pupillary area. The sensation in the lids was described as that of red hot emery particles. Sleepless nights come with the advent of hot weather and dark glasses are worn constantly during the summer to relieve the photophobia. A diagnosis of granular lids had been made previously and yellow oxide of mercury prescribed. The condition subsided with the advent of cold weather. The term granulated lids did not alarm the patient, who was unaware of the malignant character of the disease. Further relapses were attributed to the intense sunshine.

Many people hold the term "granulated lids" to be synonymous with a blepharitis in which the small incrustations along the lid margin appear as "granules" somewhat similar to small pieces of "granulated" sugar. The term conveys little to the patient as to the seriousness of the disease. The profession would be well advised to abandon entirely the terms "granular" and "granulated" lids. It is to be urged that the term trachoma only, should be used to patients and the malignant and communicable character of the disorder emphasized.

One of the most important steps towards the eradication of trachoma amongst the Indian people will be education. The fatalistic conception that, once the disease has been established, nothing can be accomplished other than awaiting the inevitable outcome, must be offset. Considering the mentality of these people, the results to date have well justified the efforts expended. Special attention is given to the older Indian and Medicine Man who is 
encouraged at clinics to note the manifestations and ravages of the disorder. Demonstrating a few children with normal eyelids together with a second group of positive cases who come from families where the disease has wrought havoc with vision, conveys the idea of the communicable nature. The abundant masses of soft gelatinous follicles in the lids of this group are drawn to their attention. These people realize the communicable character of diseases like small-pox, but had little conception that trachoma was of a similar nature. The importance of fingers, hands, towels and wash basins, in spreading the disease is emphasized.

Improvements are frequently noted on a return visit to an area where many of the positive cases have since provided their own toilet articles.

The old women are much more intractable in this respect than the older men. They view with suspicion any suggestions coming from a stranger and are inclined to scoff at anything savouring of advice. Other than through the influence of the husband, it will be most difficult to enforce the discontinuance of the practice of wiping the children's faces with the end of the head kerchief which is constantly used to mop the secretion from her own diseased eyes. Much less difficulty will be experienced with the younger mothers who have had an opportunity of training in the residential schools.

The residential school should prove to be a most valuable asset in educating the rising. generation to the danger of the disease and its manner of propagation. The importance of early and consistent treatment is learned by the pupils, many of whom are under treatment. Extreme care is exercised to render treatment as gentle and painless as possible.

Criticism of this policy is justifiable on the basis that these cases are a grave menace to the non-infected pupils, and should never have been admitted to these institutions. The ideal would undoubtedly be trachoma-free schools. Returning these children to their homes would result in their securing little medical attention and the proper nutrition which is so necessary to supplement the former.

The experience of other nations has demonstrated that once trachoma has been introduced to any great degree, the process of extermination has been measured in terms of years and not months. Since it has obtained such a foot-hold in the Canadian Indians, there is little evidence to presuppose that the disorder will be of any shorter duration. On the contrary, in addition to other factors, there must be overcome certain fundamental prejudices which are inherent in the Indian nature. The pupils of today will become the parents of tomorrow, and it is most essential that they secure enlightenment regarding the disorder. The school is the natural and most advantageous place from which to spread all propaganda. 
The common roller towel has been eliminated from these schools and each child has an individual towel, basin, and soap. The positive cases have their pillow sheets, towels, etc., marked with a large square of turkey-red unbleachable cotton percale. Strict discipline is maintained regarding the use of toilet articles. Presupposing infection during play hours in the late afternoon, each child who is negative receives prophylactic drops of zinc sulphate one-quarter per cent. ( 0.25 per cent.) each evening. This measure has proved quite effective to date. In addition to any other treatment all positive pupils receive at the same period trachomine ointment. Towels, handkerchiefs, etc., from the infected pupils are boiled separately for 20 minutes before being laundered. Antiseptics such as creolin, lysol, etc., and their derivatives are discouraged forr laundry purposes as there is always a danger of over-confidence in their value. The extremely alkaline reaction of the water through chemical interaction seriously interferes with their antiseptic properties. Special regulations have been laid down with respect to the bathing of the trachoma patients. Every effort is made to protect the uninfected individual from accidentally contracting the disorder. To date the regulations, wherever carried out, have proven quite satisfactory. Cases developing a corneal ulcer with consequent marked lacrymation with high index of infectivity, are especially guarded against.

It might be of interest to discuss briefly the possible origin of trachoma in the Canadian Indians. Many of the more intelligent and older members of the Stoney and Cree nations believe the disease was introduced by infected Indians from the United States who accompanied the early traders from the American side into the southern parts of the Prairie Provinces and British Columbia. Many of these visitors remained and were absorbed into the Western bands. Some of the more responsible Indians claim this to be the manner in which trachoma was introduced to their people.

That the Indians might have contracted the disorder from the earlier white settlers may be possible but is highly improbable. Many of these foreigners did not arrive in the country until after 1890 , and it is scarcely conceivable that the disease could become so generalized throughout the West in such a limited period. Many of the older infected Indians maintain this eye trouble was present in their own families with the resultant blindness long before the advent of the European settler.

Certain authorities claim that the American Indians were originally a group of nomad races from the plains of Mongolia, who crossed to the American continent in various huge migrations. Should this be true and one considers the incidence of trachoma in the Mongols, it is quite possible that the Indians have had 
the disorder from time immemorial. The presence of the disease in large numbers of people in the hinterlands of British Columbia could well be accounted for on these premises, as little fraternizing is encountered between the Indians to the east of the Rocky Mountains and those living on the western and Pacific side. It is scarcely possible that one or other of these large groups of Indians differing entirely in mode of life, customs and language, would be responsible for the disorder in the other group. Quite compatible with the possible Asiatic origin of trachoma is the fact that many of the Indians held a fatalistic and stoical conception of the ultimate results to be expected in one who had the disease. From all observations they have no drugs of any value against the malady and the condition was permitted to run a course devoid of even primitive medication. A long experience and contact with the disorder would cause them to view the whole process from such a fatalistic standpoint. Many old people who have lost the major portion of their sight years ago, assert that their immediate parents and grandparents were afflicted in a similar manner. At present the best evidence would indicate that trachoma has been present in these people for generations.

Certain observations might prove of interest.

(a) The familial character of trachoma. The chief source of infection and the great channel of transmission is through the family. Examination of children coming from various districts in the residential school invariably indicates the families and areas where one can be certain to encounter the disorder in the greatest degree. In such an area one notes either mental lethargy or stupid stoicism in the attitude of the inhabitants toward the disease. They either see or do not see, and many do not recognize any gradation lying between these opposite poles. This may be due possibly to the insidious onset and imperceptible continuity of the process.

(b) Certain cases tend to be more resistant to treatment where an accompanying scrofulous diathesis exists. Production of scar tissue by various methods of stimulation has a much poorer response than in more healthy individuals.

(c) Climate and local factors have an important bearing on the incidence and severity of cases. Areas with high altitude where fine alkali dust, frequent dust and sand storms, intense dryness and heat exist, have the highest index. Here also are encountered the most extreme cases. Districts such as Northern Manitoba or Alberta and in the coastal region of British Columbia where there is more abundant rainfall and much green foliage appear to have a much lower incidence. Positive cases have a much more benign character. It is highly improbable that the more exclusive fish diet of the lower areas as opposed to the high meat consumption in the trachoma belts, has any appreciable influence on the condition. 
(d) Certain individuals in "trachoma" families appear to have an inherent immunity to the disorder. There is little reason to presuppose that they had not been repeatedly infected as no undue precautions have been instituted to protect themselves against others within the same household.

(e) Some cases become quiescent with the history of total absence of treatment. The lid condition shows no signs of any activity and casual examination of the pannus shows a relative anaemia. The blood vessels in the area are noted with the 8 magnification glass. How treacherous is this condition only observation and time will tell. Such cases are classified as "arrested."

$(f)$ Trachoma does not run a steadily progressive and destructive course. Periods of exacerbations are interpolated between those of the dormancy of sub-chronicity. Many cases of natural "arrest" may prove to be only manifestations of a dormant state. Limited observations to date would appear to confirm this.

In conclusion it may be stated that every effort is being expended compatible with available finances, to bring the situation under control. Dr. E. L. Stone, Director of Medical Service, of the Department of Indian Affairs, is to be commended for instituting the campaign which, it is hoped, will ultimately eradicate trachoma in the Indians of Canada. In this connection, it is most important to keep enthusiasm alive and be prepared to be undeterred by apparent regressions in certain areas. The characteristic chronicity of the disorder will only be overcome by persistent effort. Education of the rising generation with subsequent co-operation on their part, will undoubtedly prove the most potent factor in the solution of the trachoma problem in their people.

\title{
OPHTHALMOPLEGIA ASSOCIATED WITH BONY CHANGES IN THE REGION OF THE SPHENOIDAL FISSURE
}

BY

\author{
Charles B. V. Tait
}

LONDON

My reason for recording the details of this case is twofold. First because uncertainty must attain as to the nature of the lesion and secondly, in spite of the very extensive nature of this lesion and the gravity of the prognosis, the patient has made an almost complete recovery.

M. S., a schoolgirl, aged 17 years, had been referred to $\mathrm{Mr}$. 